\title{
STATIC TESTING OF AN INFLATABLE/RIGIDIZABLE HEXAPOD STRUCTURE
}

\author{
K.T.BERGER, Virginia Polytechnic Institute and State University \\ L.G.HORTA, Structural Dynamics Branch, NASA Langley Research Center \\ B.K.TALEGHANI, U.S. Army Vehicles Technology Directorate
}

\begin{abstract}
Scientific application of large space antennas and telescopes is fostering the study of ultralightweight and inflatable structures. These socalled "gossamer" structures are tightly packaged for launch and subsequent deployment. Of particular interest is a hexapod configuration often found in vehicle ride simulators, ground-based telescopes, and antennas. One such configuration was modeled and tested at NASA Langley. Discrepancies between dynamic test data and predictions from a finite element model prompted additional static testing. This paper discusses static tests that were conducted to update stiffness parameters in the finite element model.
\end{abstract}

\section{INTRODUCTION}

There is a growing interest in large lightweight structures for space use in communication antennas, solar power concentrators, and solar sails for propulsion. Engineering of such systems presents a tremendous challenge to achieve designs within weight, volume, and cost constraints. With structure sizes exceeding 100 meters, one approach is to use multiple smaller, lightweight, inflatable structures flown in formation to function like a large system. For example, a large parabolic mirror can be approximated using multiple flat membranes each collecting and focusing light onto a remote small conventional mirror. In a configuration like this, the small flat membranes are easier to fabricate and less expensive to transport into space. Ultra lightweight structures can be packaged in small containers, and once in space, inflated using pressurized gas. Once deployed, rigidization is achieved through the cooling of thermoplastic resins in the structure and pressure is no longer required.

The hexapod test article, shown in Figure 1, is a generic system built to study this class of structures. To test such a system, conventional instrumentation like accelerometers, greatly increases the mass of the structure, and thus significantly alters its static and dynamic responses. Instead, non-contact measurement techniques such as photogrammetry are more appropriate ${ }^{1}$. Photogrammetry (a method to recover 3D geometry from photographs) is used to measure displacements with no significant impact on the mass or stiffness. The results of the photogrammetric tests are discussed in the paper.

\section{DESCRIPTION OF TESTBED}

Figure 2 shows a view of the hexapod in the direction perpendicular to the torus plane. As shown, the torus segmented construction has twelve 0.181-meter diameter tubes arranged to form a circle. Six tapered struts, with diameters

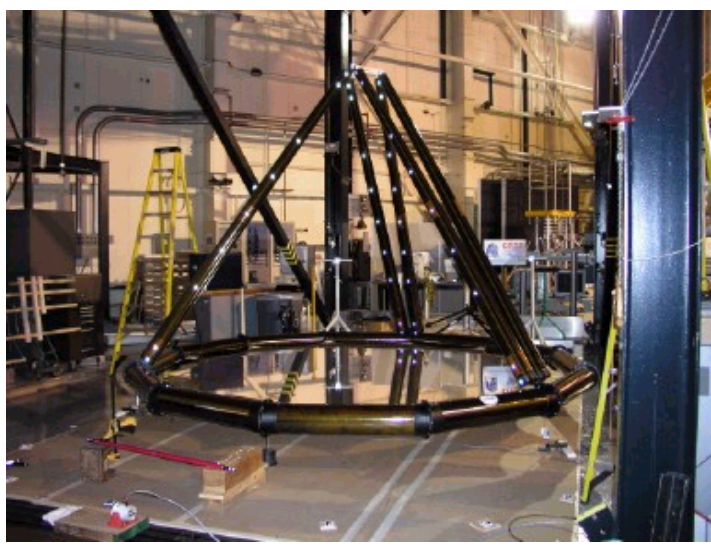

Figure 1: Hexapod loaded in test configuration

ranging from 0.0795 to 0.130 meters, connect the torus to a triangular aluminum frame. Tubes for the torus and struts are fabricated using a proprietary thermoplastic graphite epoxy composite, developed by ILC-Dover, Inc. When this material is heated above the glass-transition temperature of the thermoplastic epoxy, its stiffness decreases enough to allow it to be folded 
or rolled, and thus packaged into a much smaller volume $^{2}$. Rigid joints connecting the sections of the torus are cast from glass filled urethane. A Kapton membrane, with a vapor deposited aluminum coating $25.4 \mu \mathrm{m}$ thick, is stretched within the torus. The structure was rigidized prior to its assembly.

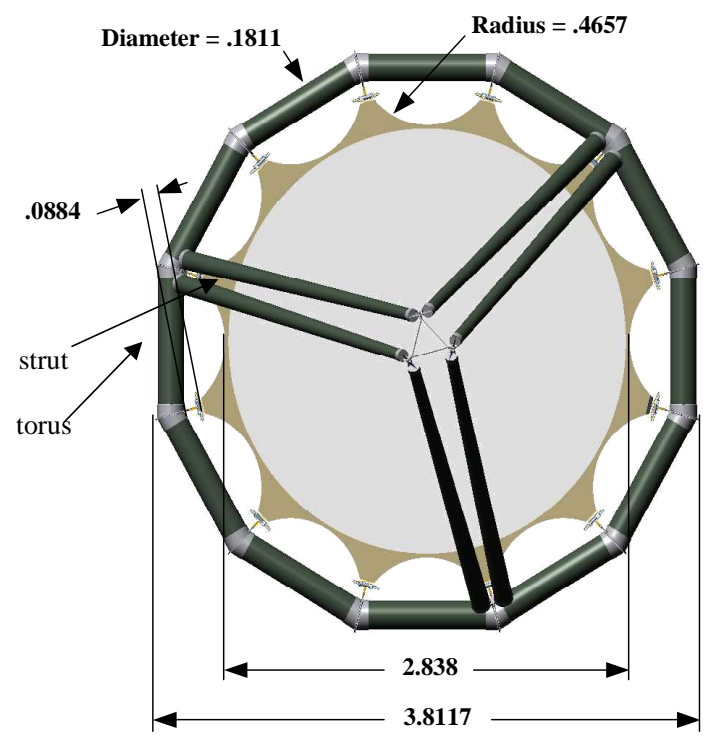

Figure 2: Dimensions of Hexapod in Meters

\section{STATIC TEST SETUP}

To facilitate the static tests, the hexapod was moved from its original vertical orientation to a horizontal orientation, with the membrane and torus parallel to the floor, as shown in Figure 1. A force is applied perpendicular to a urethane joint on the torus (shown in Fig. 3), and displacement measurements were taken along the circumference of torus. Load cells placed in-line between the torus and the floor are used to measure support loads. The locations of the applied force and the load cells are shown in Figure 3. All loads are applied and measured in the direction perpendicular to the plane in Fig. 3. In the first set of tests, only load cells 2, 4 and 5 are used. Later, additional supports with in-line load cells 3 and 6 are added to the system to reduce rotation of the torus during loading. The applied load used for the tests was $42.7 \mathrm{~N}$.

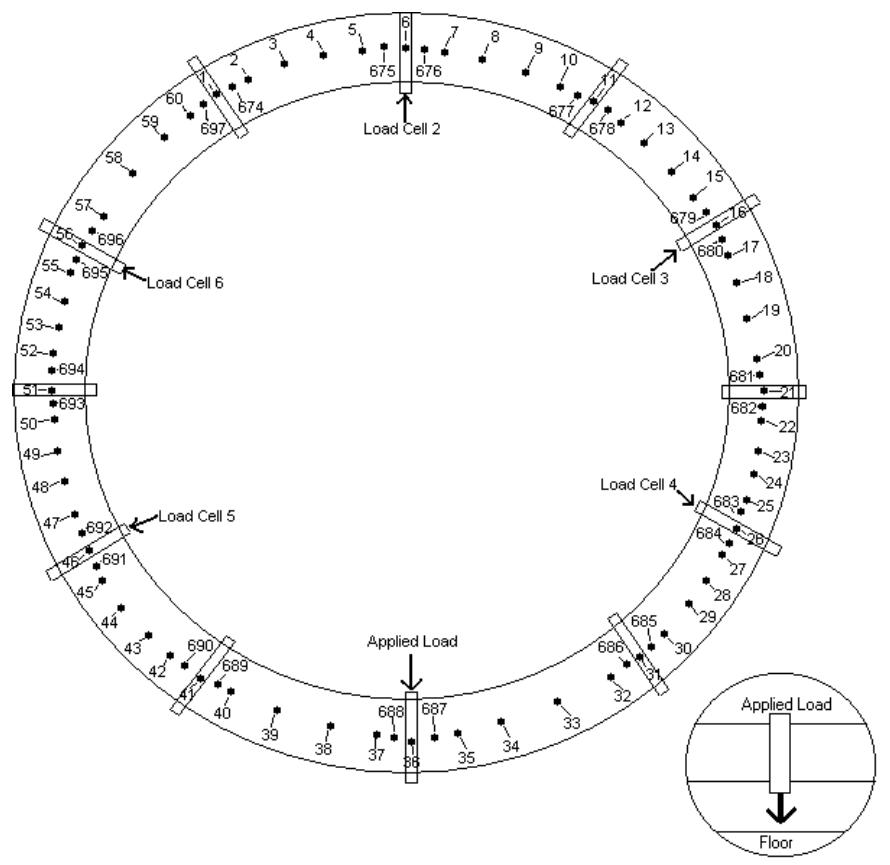

Figure 3: Locations of Targets, Load Cells and Applied Load

Two model SM50 load cells, manufactured by Interface, Inc., are used to measure boundary loads and to support the torus in the horizontal configuration. These load cells can measure up to $222.4 \mathrm{~N}$ in the tension and compression directions. Figure 4 shows that the in-line load cell and support system consists of a metal block into which a bolt on the support strut is set. The bolt on the support strut is attached to the bottom of the load cell, another bolt is attached to the top, and a washer is welded onto the top bolt, which attaches to the hexapod urethane joint.

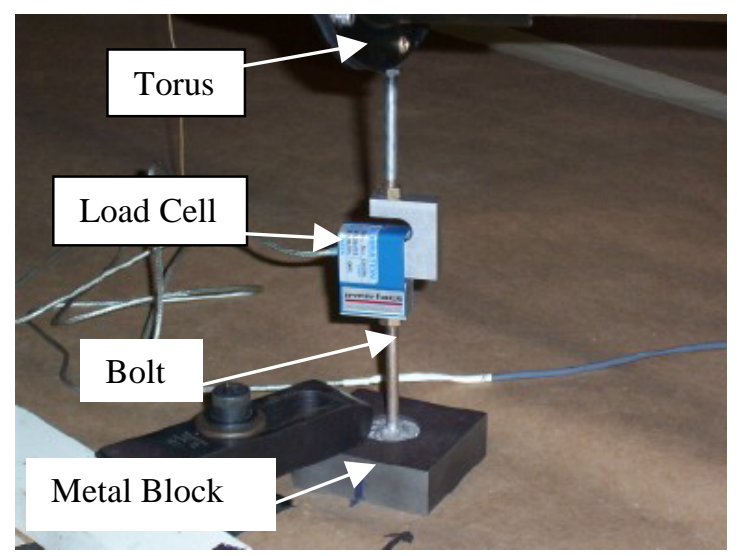

Figure 4: Load Cell/Support System 


\section{PHOTOGRAMMETRY}

Photogrammetry is a non-contacting measuring technique that requires little or no mass loading of the test article, thus ideal to study ultra-lightweight structures. Measurement quality of this technique is a function of our ability to photograph structural features. To enhance contrast during photograph analysis, retro-reflective targets $(9.525-\mathrm{mm}$ diameter) are placed on the torus to coincide with the nodes in the finite element model. Target locations and numbering scheme are depicted in Figure 3. Pictures are taken in the unloaded and loaded static cases for the three- and five-load cell support configurations.

A V-STARS Photogrammetry System ${ }^{3}$ is used for data analysis. Coded targets, small plates with eight retro-reflective targets placed in a unique pattern, are positioned at various control points on the floor below the torus. An INCA 6.3 17-mm camera captured 75 to 110 images for each load condition, from a variety of locations around the circumference of the torus, and from a variety of angles above the structure. These are then loaded into the software to match the coded target locations across multiple images and create an initial map of their positions. Using this initial information, the software matched the individual targets on the torus from each image to the corresponding targets from the other images. This referencing allowed the software to triangulate in 3-D space the locations of each retro-reflective target. Two scale bars are used to ensure that the scale is the same for each data set. The origin of the measurement coordinates is selected at the center of the membrane. From the computed 3-D target locations, the geometry is created for each of the load cases and compared to geometries for the other load cases, allowing the deflections due to loading to be determined.

After the data was collected, the unloaded case was examined more closely and checked for points exhibiting large error values. Obstructed targets from one photo to the next often caused most of the large errors. As a minimum, each target must be seen, marked, and referenced in 2 images, but for greater accuracy more images are best. Camera angles greater than 30 degrees are recommended to accurately triangulate the coordinates of each target.

After the unloaded case was processed, the same procedure was followed for the loaded three-load cell and loaded five-load cell configurations. It was found that, in general, targets located on the torus joints that were attached to the struts were harder to see. High measurement errors were attributed to camera views where the struts blocked the view, thus decreasing the number of images in which those points appeared.

A LK-501 Laser Displacement Sensor was used to verify the photogrammetric results. The laser was placed directly over a selected point and the deflections in the $\mathbf{Z}$ direction (gravity direction) were recorded for a number of different loads. The process was repeated at various locations and compared to the photogrammetric measurements.

\section{BOUNDARY STIFFNESS ESTIMATION}

The load cell/support system (Figure 5) was tested to determine the boundary interface stiffness. This interface stiffness is modeled as a translational spring in the finite element model ${ }^{4}$. One load cell was removed from the system and a small bracket was placed on top. It would have been preferable to leave the load cell attached to the hexapod structure to test the entire system, but the displacements observed were so small that accurate measurements with the laser could not be obtained. A stinger was attached to the top of the load cell/support system, parallel to the floor and used as target for the laser displacement sensor. Various weights were placed on top of the bracket and the corresponding deflections measured using the Laser Displacement Sensor.

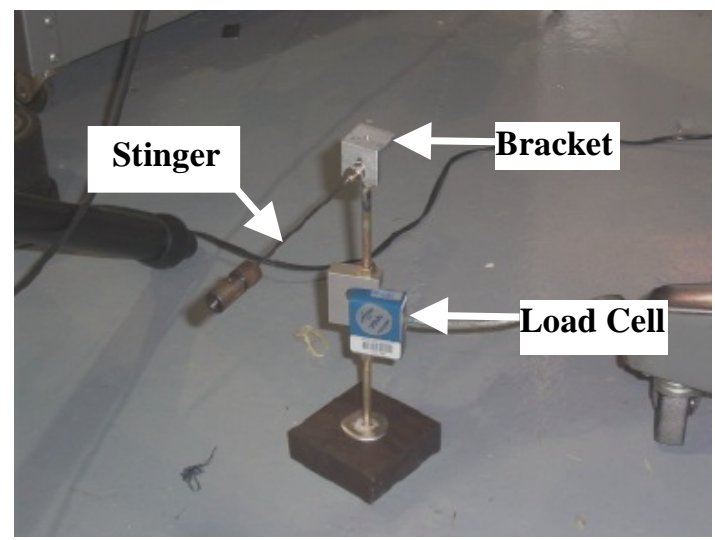

Figure 5: Load Cell/Support system with stinger and bracket attached

Combining the displacement data and the known forces applied, a stiffness value was estimated for the load cell/support system. The data shown in Figure 6 was used to curve fit the stiffness value of the load cell/support system to be $1024 \mathrm{KN} / \mathrm{m}$. 


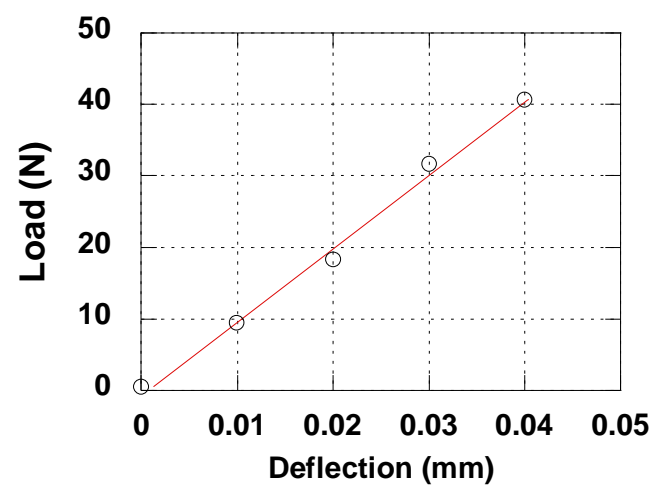

Figure 6: Support stinger load deflection data

\section{DISCUSSION OF STATIC TEST RESULTS}

Static deflection tests were performed on the torus section of the hexapod structure only. Data for various load cases with the torus supported at three and five load cells can be seen in Figures 710. Figure 7 is for the unloaded structure with three supports while Figure 8 is loaded with 42.7 N. Similarly, Figure 9 is for the unloaded structure with five supports while Figure 10 is loaded with $42.7 \mathrm{~N}$.

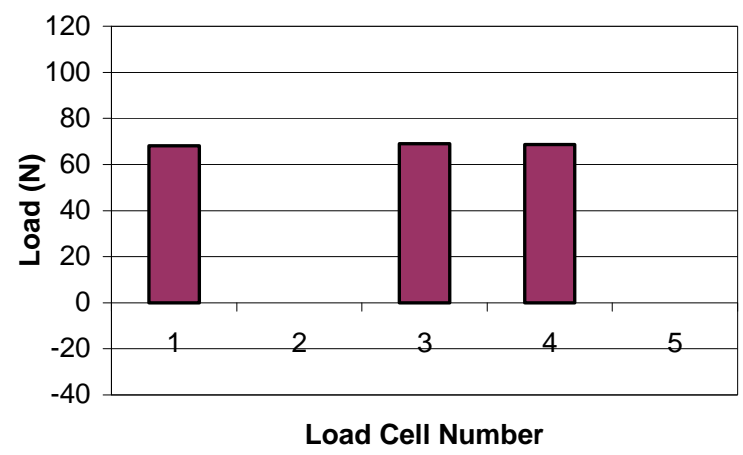

Figure 7: 3-Load Cell Configuration Unloaded

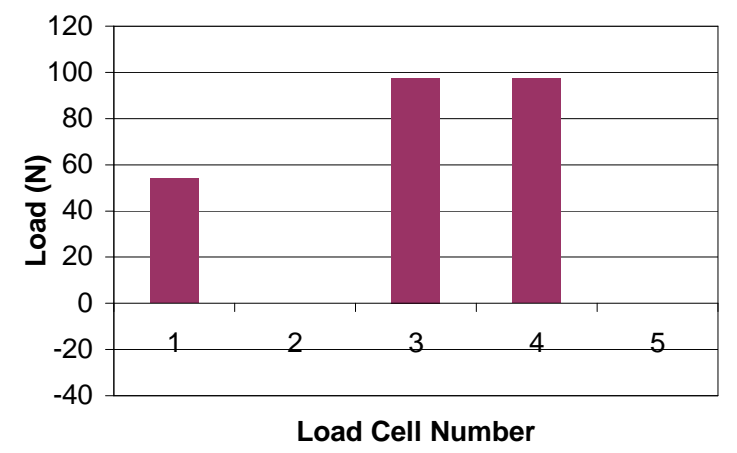

Figure 8: 3-Load Cell Configuration Loaded with $42.7 \mathrm{~N}$

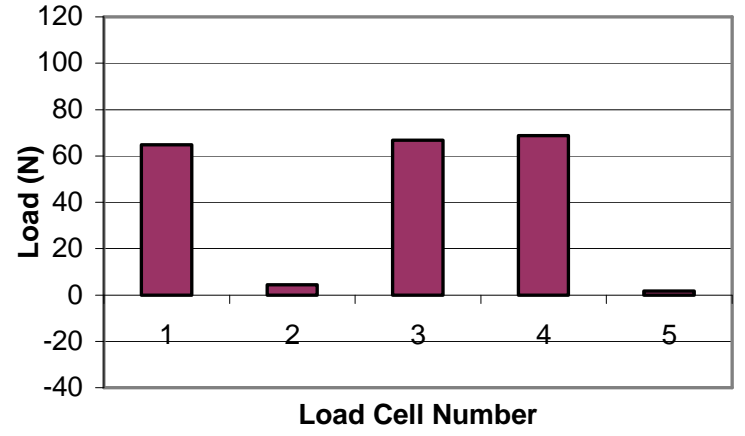

Figure 9: 5-Load Cell Configuration Unloaded

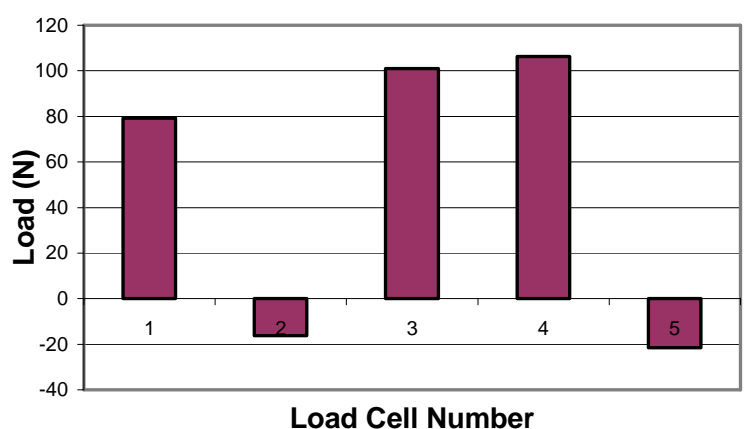

Figure 10: 5-Load Cell Configuration Loaded with $42.7 \mathrm{~N}$

When the loaded cases were compared with the unloaded case, the largest displacements were found at the point where the force was applied at Node 36 (Figure 3). The majority of the movement was in the $Z$ direction, with only minimal movement of the structure in the $X$ and $Y$ direction (always less than $0.5 \mathrm{~mm}$, and primarily less than $0.2 \mathrm{~mm}$ ). With the exception of a small number of hard to see target points in each data set, the results show smooth deformations in the $Z$ direction between the loaded and unloaded cases for three- and five-load cell configurations.

As shown in Figure 11, for the three-load cell configuration, the maximum deflection was found to be $4.075 \mathrm{~mm}$, located at Node 36 . There was significant rotation and bending of the structure, as shown in the V-STARS diagram of the residuals in the $Z$ direction. The residuals were magnified to 200 times their actual length for ease of viewing. Displacements in the $Z$ direction around the torus varied from $1.554 \mathrm{~mm}$ at Node 21 to $1.245 \mathrm{~mm}$ at Node 52. 


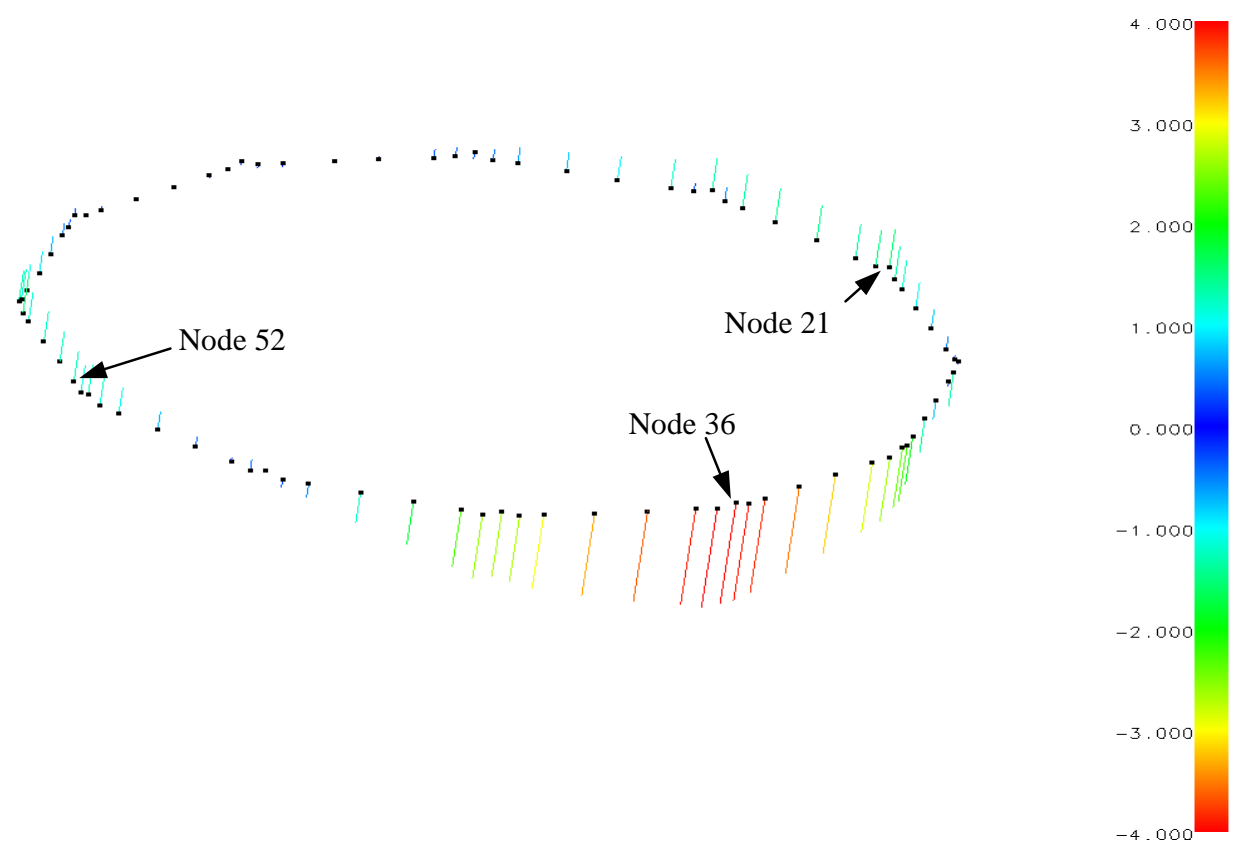

Figure 11: Photogrammetry displacement data for 3-load cell configuration

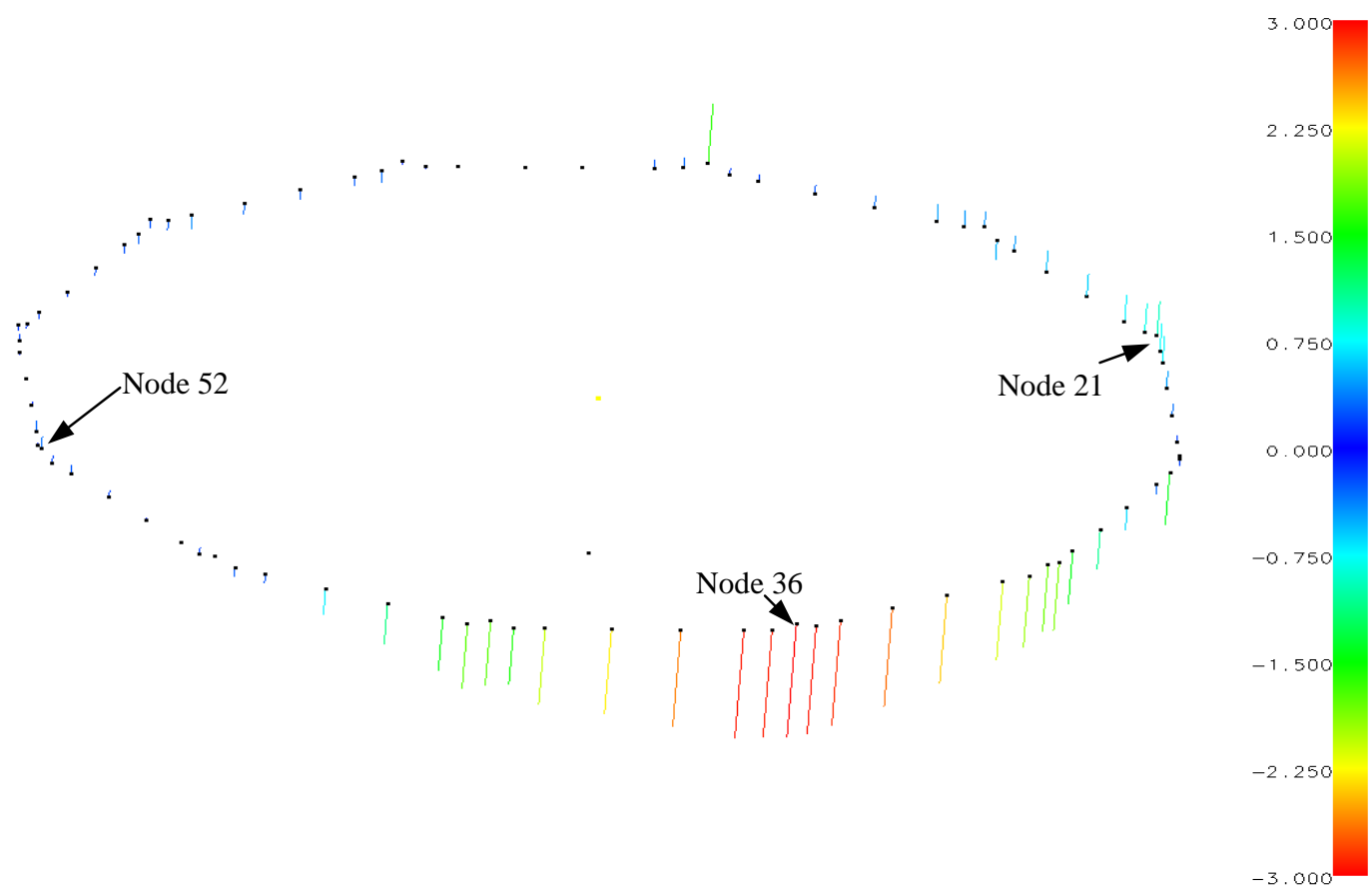

Figure 12: Photogrammetry displacement data for 5 -load cell configuration

Because of the large rotation of the structure, two additional support points with corresponding load cells were added to the system to make the torus more rigid. The displacements in the $\mathrm{Z}$ direction for the five-load cell case are shown in Figure 12 Comparing the loaded case to the unloaded case for the five-load cell configuration, a smaller maximum displacement in the $Z$ direction was found. This result was expected, as the structure was being supported at more locations than in the previous three-load cell configuration. The maximum displacement for this case was 3.054 $\mathrm{mm}$, again located at Node 36 . While there is still some rotation of the structure, it is significantly 
less than the previous case, at $0.933 \mathrm{~mm}$ at Node 21 and $0.321 \mathrm{~mm}$ at Node 51.

A graph comparing the out-of-plane $Z$ displacements of the three and five load cell configurations is shown in Figure 13. They are graphed using the node numbers 1-60, with the

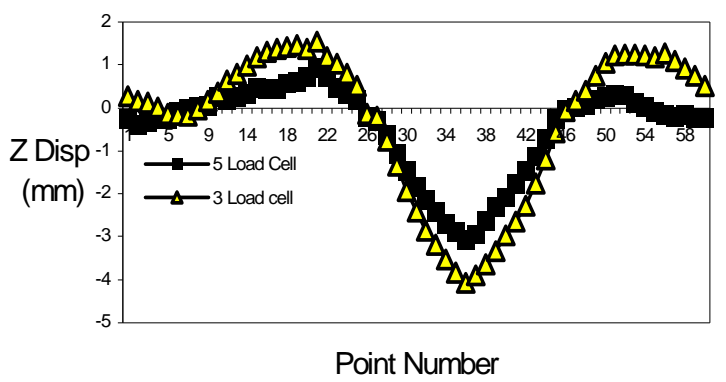

Figure 13: Delta $Z$ as a function of point number

additional points (674-697) located in the correct positions between Nodes 1 and 60 . There is significantly less rotation and movement for the structure when five load cells are used.

\section{CONCLUDING REMARKS}

The tests discussed here were designed to determine vertical displacements of the torus section of a hexapod test article under load conditions. Results from photogrammetric measurements show the ability to use this measurement technique to test this class of structures with no adverse impact from the instrumentation. It was determined that the measurement resolution is adequate to characterize the static behavior of these lightly loaded systems. Boundary supports need to be carefully designed and characterized if data is to be used in a model update process.

\section{ACKNOWLEDGMENTS}

The authors would like to thank Richard Pappa and Jonathan Black for their help with the photogrammetric measurements, as well as Mercedes Reaves for her help with the finite element model.

\section{REFERENCES}

1. Pappa, R.S., Jones, T.W., Black, J.T., Walford, A., Robson, S. and Shortis, M.R., "Photogrammetry Methodology Development for Gossamer Spacecraft," NASA/TM-2002-211739, June 2002

2. Adetona, O., Keel, L.H., Horta, L.G., Cadogan,
D.P., Sapna, G.H., and Scarborough, S.E. "Description of new inflatable/rigidizable hexapod structure testbed for shape and vibration control", Paper \# AIAA-2002-1451, Proc.43 ${ }^{\text {rd }}$ AIAA structures, structural dynamics and materials conference, Denver, CO, April 22-25, 2002.

3. Geodetic Services, Inc., Melbourne, FL, VSTARS Industrial Photogrammetry Systems, www.geodetic.com

4. Adetona, O., Horta, L.G., Taleghani, B.K., Blandino, J.R., Woods, K.J., "Vibration Studies of an Inflatable/Rigidizable Hexapod Structure with a Tensioned Membrane," Paper \# AIAA-2003-1737. 